\title{
Home-based care: Implications for education and insurance providers
}

Abstract. Many papers have been published that concern technological change and its application to health service delivery. However, this is just one of the innovations required to establish a holistic solution for a purposeful intervention. A further two are considered in this paper: education provision to support all stakeholders involved in the health delivery system; and insurance-led services to pay for some of the support required. The transition of point-of-care delivery from hospital-based care to home-based care is also a focus of the work. Key adoption issues for the change are identified, from which implications for the education and insurance sectors are discussed. Gaps in provision are recognized and outline solutions suggested. It is clear that there are beacons of good practice in the areas scrutinized, but it is found that it is sometimes difficult to disseminate findings and scale up provision from the NHS whole-system demonstrators in eHealth.

Nanotechnology Perceptions 13 (2017) 61-68

doi: 10.4024/N03SU17A.ntp.13.01 